\title{
Possible involvement of glutathione balance disruption in dihydropyrazine-induced cytotoxicity on human hepatoma HepG2 cells
}

\author{
Takumi Ishida, Shinji Takechi and Tadatoshi Yamaguchi \\ Faculty of Pharmaceutical Sciences, Sojo University, Ikeda 4-22-1, Nishi-ku, Kumamoto 860-0082, Japan
}

(Received May 23, 2012; Accepted July 21, 2012)

\begin{abstract}
Dihydropyrazines (DHPs), formed by nonenzymatic glycation, are known to exert various effects in vitro and in vivo, such as generation of radical species, DNA strand breakage, enzyme inhibition, and inhibition of bacterial growth. However, their effects on mammalian cells remain elusive. To address this issue, we investigated the effects of a range of DHP concentrations on human hepatoma HepG2 cells using 2,3-dihydro-5,6-dimethylpyrazine (DHP-1), 2,3-dihydro-2,5,6-trimethylpyrazine (DHP-2), and 3-hydro-2,2,5,6-tetramethylpyrazine (DHP-3) as model compounds. All of the tested compounds exerted cytotoxic activity against HepG2 cells in the range of $10 \mu \mathrm{M}-1 \mathrm{mM}$, and significantly so at the highest concentration. DHP-3 was the most effective drug, and it also caused a significant decrease in the ratio of intracellular reduced and oxidized glutathione (GSH/GSSG). In addition, the cytotoxic effect of DHP-3, but not DHP-1 and DHP-2, was enhanced by the inhibition of GSH biosynthesis using $100 \mu \mathrm{M}$ L-buthionine-(S,R)-sulfoximine (BSO). From these results, it is suggested that the mechanisms of cytotoxicity exerted by DHP-3 are distinct from those exerted DHP-1 and DHP-2. In addition, it is possible that the disruption of intracellular glutathione balance induced by DHP-3 is related to its effect on HepG2 cells.
\end{abstract}

Key words: Dihydropyrazine, Glutathione balance, HepG2 cell, Diabetes

\section{INTRODUCTION}

Dihydropyrazines (DHPs) produced by the condensation of 2 molecules of D-glucosamine are intermediates of the glycation reaction (Kashige et al., 1995). Since many pyrazine derivatives, which are deduced metabolites of DHPs, have been found in human urine (Zlatkis et al., 1973) and various foods (Maga, 1982; Joo et al., 1997), it is speculated that DHPs would universally exist in the human body and food. A number of studies have addressed the biological effects of DHPs. Teixeira et al. (2001) reported that 3,6-dihydropyrazine-2,5-dipropanoic acid, the cyclic product derived from the condensation reaction of 5-aminolevulinic acid, acted as a DNA strand-breaking and oxidizing agent. We also reported that methyl-substituted DHPs (Me-DHPs) have DNA strand-cleaving activity (Yamaguchi et al., 1996), generate hydroxyl and carbon-centered radicals (Yamaguchi et al., 2012), inhibit growth and induce mutagenesis of Escherichia coli (Takechi et al., 2004; Takeda et al.,
2005), and induce damage to cellular proteins such as glyceraldehyde-3-phosphate dehydrogenase (Takechi et al., 2010). Phenyl-substituted DHPs (Ph-DHPs) have higher DNA strand-cleaving activity and potency to generate radical species than do Me-DHPs (Yamaguchi et al., 1999). However, the sensitivity to DNA repair-deficient bacteria induced by Ph-DHPs is lower than that induced by Me-DHPs (Takeda et al., 2007). In addition, cyclohexyl-DHP, which was designed according to our hypothesis of a relationship between the chemical structure of DHP derivatives and their DNA strand-cleaving activity (Maruoka et al., 2005), was shown to possess stronger DNA strand-cleaving activity than Me-DHP or Ph-DHP (Yamaguchi et al., 2007). More recently, it has been reported that the reaction of some Me-DHPs or cyclohexyl-DHP with GSH is a potential mechanism of bacterial growth inhibition (Takechi et al., 2011).

In spite of all the available data, the biological effects of DHPs on mammalian cells remain elusive. To address this issue, we studied the effects of DHPs on human

Correspondence: Takumi Ishida (E-mail: ishida@ph.sojo-u.ac.jp) 
<smiles>[R]C1([R])CN=C(C)C(C)=N1</smiles>

Fig. 1. Chemical structures of the dihydropyrazines (DHPs) used in the present study. 2,3-Dihydro-5,6-dimethylpyrazine (DHP-1): $\mathrm{R}_{1}=\mathrm{H}, \mathrm{R}_{2}=\mathrm{H} ; 2$,3-dihydro-2,5,6trimethylpyrazine (DHP-2): $\mathrm{R}_{1}=\mathrm{CH}_{3}, \mathrm{R}_{2}=\mathrm{H}$; and 3-hydro-2,2,5,6-tetramethylpyrazine (DHP-3): $\mathrm{R}_{1}=$ $\mathrm{CH}_{3}, \mathrm{R}_{2}=\mathrm{CH}_{3}$.

hepatoma HepG2 cells using 2,3-dihydro-5,6-dimethylpyrazine (DHP-1), 2,3-dihydro-2,5,6-trimethylpyrazine (DHP-2), and 3-hydro-2,2,5,6-tetramethylpyrazine (DHP3) (Fig. 1) as model compounds. In addition, to understand the mechanisms exerted by DHPs, we focused on the changes in intracellular reduced and oxidized glutathione (GSH) levels.

\section{MATERIALS AND METHODS}

\section{Cells and reagents}

The human hepatoma cell line HepG2 (JCRB1054) was obtained from the Human Science Research Resources Bank, Osaka, Japan. The DHPs employed were synthesized by condensation of diketones and diamines using the method by Yamaguchi et al. (1996). All other reagents and chemicals were of the highest grade commercially available.

\section{Cell culture}

HepG2 cells were cultured in Dulbecco's modified Eagle's medium supplemented with 10\% (v/v) fetal bovine serum, in a humidified atmosphere with $5 \% \mathrm{CO}_{2}$ at $37^{\circ} \mathrm{C}$. HepG2 cells were grown in $60 \mathrm{~mm}$ culture dishes to $80-90 \%$ confluence before being subject to various treatments.

\section{Cell viability assay}

DHP stock solution was prepared by dissolving in dimethyl sulfoxide (DMSO) at $1 \mathrm{M}$, and this was stored at $-20^{\circ} \mathrm{C}$ until use. A stock solution was diluted with DMSO to give concentrations of 1,10 , and $100 \mathrm{mM}$, which were then each diluted 10 times with distilled water. Each prepared DHP reagent was diluted 100 times with culture medium. As a result, the final concentrations of DHP and DMSO were adjusted at $1,10,100$, and $1,000 \mu \mathrm{M}$ (DHP) and $0.1 \%(\mathrm{v} / \mathrm{v})(\mathrm{DMSO})$, respectively. Cell viabil- ity was determined by using the Cell Count Kit-8 (Dojin Molecular Technologies Inc., Kumamoto, Japan) according to the manufacturer's instructions. Briefly, HepG2 cells were seeded at a density of $1 \times 10^{5}$ cells/well in a 96-well plate. After $24 \mathrm{hr}$, the culture medium was replaced with DHP-containing medium. Cells were exposed to chemicals for $24 \mathrm{hr}$, and after washing the cells with $150 \mu \mathrm{l}$ of phosphate-buffered saline (PBS), new culture medium containing $10 \%(\mathrm{v} / \mathrm{v})$ of WST- 8 dye solution was added to each well. Cells were incubated for 30-60 $\mathrm{min}$ in a $\mathrm{CO}_{2}$ incubator to develop color. The absorbance was measured at a wavelength of $450 \mathrm{~nm}$ using a microtiter plate reader. To investigate the effect of GSH biosynthesis on DHPmediated cytotoxicity, the cells were cultivated for $24 \mathrm{hr}$ in the absence or presence of $100 \mu \mathrm{M}$ L-buthionine-( $S, R)$ sulfoximine (BSO; Wako Pure Chemical Industries, Ltd., Osaka, Japan) before addition of DHP.

\section{Determination of reduced and oxidized GSH}

Reduced and oxidized GSH levels were determined with the GSSG/GSH Quantification Kit (Dojin Molecular Technologies Inc., Kumamoto, Japan) according to the manufacturer's instructions. Briefly, cells exposed to DHP for $24 \mathrm{hr}$ were obtained by scraping off the bottom of the dish with a cell scraper. The cell pellets were washed with ice-cold PBS and resuspended with $80 \mu \mathrm{l}$ of $10 \mathrm{mM} \mathrm{HCl}$. Then, they were frozen and thawed twice for cell lysis. After the addition of $20 \mu 1$ of $5 \%$ (v/v) 5-sulfosalicylic acid, the mixture was centrifuged at $8,000 \times \mathrm{g}$ for $10 \mathrm{~min}$ at $4^{\circ} \mathrm{C}$. Total GSH and oxidized GSH (GSSG) levels in the supernatant were determined according to the manufacturer's protocol. Reduced GSH levels were calculated based on the measured concentrations of total GSH and GSSG using the following formula: Reduced GSH $(\mu \mathrm{mol} / 1)=$ Total GSH $(\mu \mathrm{mol} / 1)-2 \times$ GSSG $(\mu \mathrm{mol} / 1)$.

\section{Statistical analysis}

Statistical differences between the 2 groups were calculated using the Student's $t$-test. Statistical differences among multiple groups were evaluated by one-way analysis of variance with a post-hoc test (Fischer's Protected Least Significant Difference Method).

\section{RESULTS AND DISCUSSION}

We investigated the effects of different DHP molecules on HepG2 cell viability (Fig. 2). An apparent decrease in HepG2 cell viability was not detected by treatment with DMSO alone (control). Upon treatment with DHP-1, a decrease in cell viability compared to control was 
observed at concentrations above $10 \mu \mathrm{M}$, although a significant decrease was only found at $1 \mathrm{mM}$ DHP. In contrast, DHP-2 treatment induced significant decreases in cell viability throughout the concentration range of $1 \mu \mathrm{M}-1$ mM. DHP-3 also induced significant decreases throughout the tested concentration range. However, unlike the dose-dependent effects of DHP-1 and DHP2 , the effect of DHP-3 at $1 \mathrm{mM}$ was prominently greater than that of other concentrations.

Next, to examine the redox status of cells undergoing DHP-induced cytotoxicity, we determined the intracellular GSH and GSSG concentrations. The intracellular GSH level was significantly increased after $24 \mathrm{hr}$ of exposure to $1 \mathrm{mM}$ DHP (Table 1). In contrast, the intracellular GSSG level was significantly increased only by the treatment with DHP-3 (Table 1). The GSH/GSSG ratio, as estimated from measured intracellular GSH and GSSG levels, was significantly increased upon the treatment of DHP-2. A similar trend was observed in the case of DHP-1 treatment, although a significance difference was not detected. In contrast, the GSH/GSSG ratio in DHP3-treated cells was notably decreased compared with DMSO alone. The ratio of GSH and GSSG decreases under oxidative stress conditions; thus, the ratio has been considered as a dynamic indicator of intracellular redox status (Hultbery et al., 1999; Jones, 2002). As mentioned in the Introduction, we previously reported that DHP-3 generates hydroxyl and carbon-centered radicals in vitro (Yamaguchi et al., 2012). Therefore, it seems reasonable to observe a decrease in the intracellular GSH/GSSG ratio in DHP-3-treated HepG2 cells. However, if so, there remains the question of why DHP-3 treatment did not lead to the decrease in the intracellular GSH. Regarding this point, it is possible that the disruption of glutathione balance accompanied by an accumulation of intracellular GSSG more significantly contributes to cytotoxicity of DHP-3 than oxidative stress. In fact, the decrease in GSH/GSSG ratio induced by DHP-3 was caused by the prominent increase in GSSG level rather than a change in the GSH level. This hypothesis is supported from the result shown in Fig. 3. Pretreatment of HepG2 cells with $100 \mu \mathrm{M}$ BSO, an inhibitor of GSH biosynthesis, enhanced the decrease in cell viability induced by DHP-3. It is expected that the striking decrease in intracellular GSH level produced by BSO results in the enhancement of the disruption of glutathione balance by DHP-3 compared with the situation without BSO pretreatment. Of course, the oxidative stress produced by DHP-3-derived radicals might be - at least in part_-involved in the mechanisms of DHP-3-induced cytotoxicity. However, the disruption of glutathione balance induced by DHP-3 also seems to

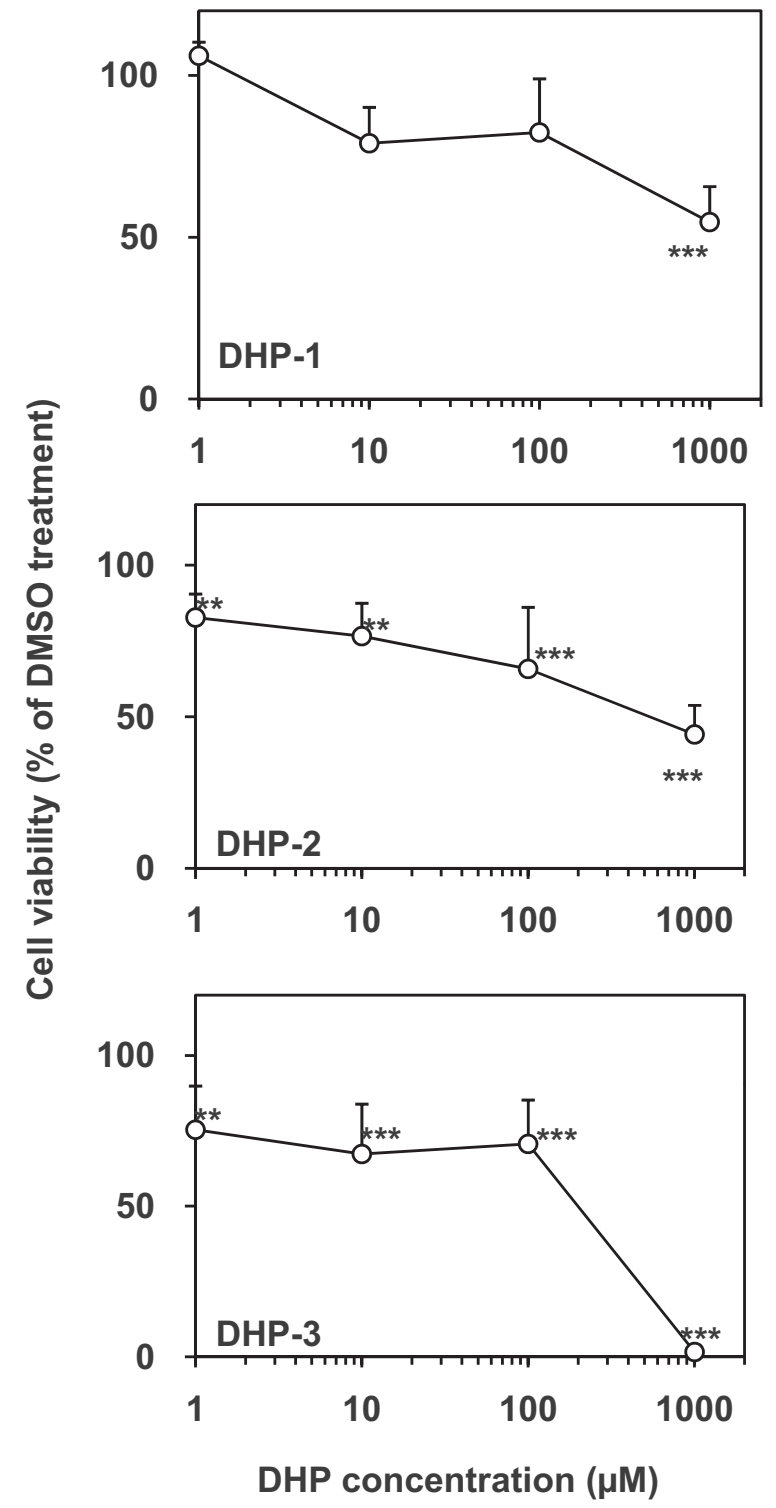

Fig. 2. Dose-dependent dihydropyrazine (DHP)-induced cytotoxicity in HepG2 cells. Cells were incubated with different concentrations of DHP-1, DHP-2, and DHP-3 for $24 \mathrm{hr}$. Control was culture medium containing $0.1 \%$ (v/v) DMSO. Cell viability was assessed by WST-8 assay. Values are represented as the mean percentage of absorbance \pm S.E. of 8 (DHP-2 at $1 \mu \mathrm{M}$ and DHP-3 at $1,000 \mu \mathrm{M}$ ) or 9 (others) independent experiments. $* * p<0.01$ and $* * * p<0.001$ versus DMSO treatment.

contribute to the cytotoxicity.

Our results showed that the changes of intracellular GSSG level and the GSH/GSSG ratio induced by DHP1 and DHP-2 were inconsistent with those from DHP-3, although they are reported to generate radical species in 
Table 1. Effects of dihydropyrazine (DHP) on intracellular GSH, GSSG, and GSH/GSSG ratio in HepG2 cells

\begin{tabular}{lccc}
\hline Treatment & $\begin{array}{c}\text { GSH } \\
(\mathrm{mmol} / \mathrm{cell})\end{array}$ & $\begin{array}{c}\text { GSSG } \\
(\mathrm{mmol} / \mathrm{cell})\end{array}$ & GSH/GSSG \\
\hline Control (DMSO) & $3.35 \pm 0.84$ & $0.06 \pm 0.04$ & $66.0 \pm 27.3$ \\
DHP-1 & $7.22 \pm 0.86^{*}$ & $0.09 \pm 0.03$ & $93.6 \pm 41.1$ \\
DHP-2 & $11.21 \pm 2.98^{* * *}$ & $0.09 \pm 0.03$ & $126.4 \pm 27.3^{* *}$ \\
DHP-3 & $8.83 \pm 2.79^{* *}$ & $0.47 \pm 0.12^{* * *}$ & $18.8 \pm 3.6^{*}$ \\
\hline
\end{tabular}

Cells were incubated with DHP $(1 \mathrm{mM})$ or DMSO $(0.1 \%, \mathrm{v} / \mathrm{v})$ for $24 \mathrm{hr}$. Cell numbers were estimated by WST- 8 assay. Values are represented as mean \pm S.D. of 5 independent experiments. ${ }^{*} p<0.05,{ }^{*} p<0.01, * * * p<0.001$ versus DMSO treatment.

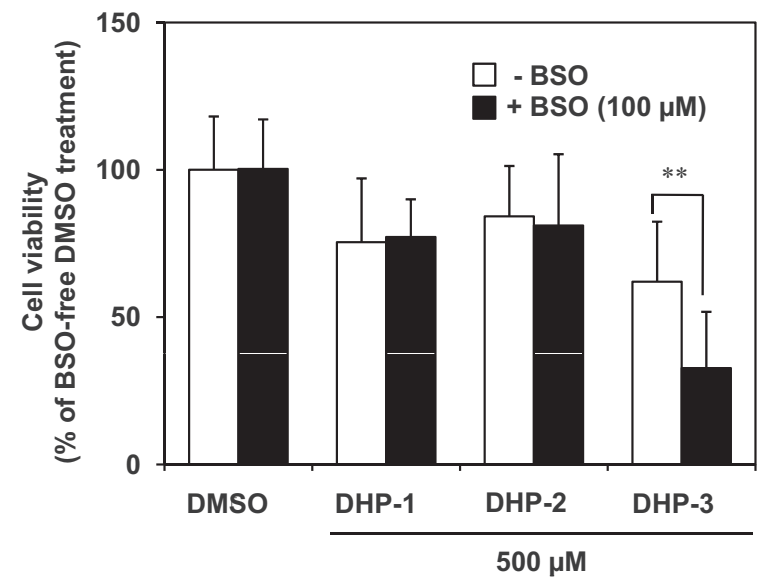

Fig. 3. Effects of the glutathione biosynthesis inhibitor, 1buthionine- $(S, R)$-sulfoximine (BSO), on dihydropyrazine (DHP)-induced cytotoxicity in HepG2 cells. Cells were incubated with BSO-containing $(100 \mu \mathrm{M})$ or BSO-free culture medium for $24 \mathrm{hr}$ prior to treatment with DHP $(500 \mu \mathrm{M})$. DMSO $(0.1 \% \mathrm{v} / \mathrm{v})$-containing medium was used as each control. Cell viability was assessed by WST- 8 assay. Bars represent the mean percentage of absorbance \pm S.E. of 8 (DHP-1 treatment without $\mathrm{BSO}$ ) or 9 (others) independent experiments. $* * p<0.01$ versus BSO-free DHP-3 treatment.

vitro (Yamaguchi et al., 2012). We were unable to clarify the mechanisms to explain this discrepancy. However, we observed that the intracellular GSH depletion by BSO treatment unaffected to the effect of DHP-1 and DHP-2 (Fig. 3). Therefore, it raises the possibility that the cytotoxic mechanisms induced by the treatment of DHP-1 and DHP-2 differ from those induced by DHP-3.

Since the concentrations of DHPs in vivo are still unclear, it remains controversial whether the concentration $(1 \mathrm{mM})$ at which DHP-induced an effect is physiologically relevant. Further studies are needed to clarify the effects of DHPs on mammalian cells, and the clarification will provide a new insight into our understanding of diseases attributed to glycation reactions such as diabetic complications.

\section{ACKNOWLEDGEMENTS}

This study was supported in part by a Grant-in-Aid for Scientific Research (C) (No. 23590160) from the Japan Society for the Promotion of Science.

\section{REFERENCES}

Hultbery, B., Anderson, A. and Isaksson, A. (1999): Thiol and redox reactive agents exert different effects on glutathione metabolism in HeLa cell cultures. Clin. Chim. Acta., 283, 21-32.

Jones, D.P. (2002): Redox potential of GSH/GSSG couple: Assay and biological significance. Methods Enzymol., 348, 93-112.

Joo, K. and Ho, C.-T. (1997): Quantitative analysis of alkylpyrazines in regular- and low-fat commercial peanut butter preparations. Biosci. Biotech. Biochem., 61, 171-173.

Kashige, N., Yamaguchi, T., Mishiro, N., Hanazono, H., Miake, F. and Watanabe, K. (1995): Possible involvement of dihydrofructosazine in the DNA breaking activity of d-glucosamine. Biol. Pharm. Bull., 18, 653-658.

Maga, J.A. (1982): Pyrazines in foods: An update. Crit. Rev. Food Sci. Nutr., 16, 1-48.

Maruoka, H., Kashige, N., Miake, F. and Yamaguchi, T. (2005): Synthesis of new dihydropyrazines with DNA strand-breakage activity. Chem. Pharm. Bull., 53, 1359-1361.

Takechi, S., Ito, S., Kashige, N., Ishida, T. and Yamaguchi, T. (2011): Glutathione depression by dihydropyrazine derivative. J. Toxicol. Sci., 36, 231-235.

Takechi, S., Nakahara, K. and Yamaguchi, T. (2010): Dihydropyrazine-induced inactivation of glyceraldehyde-3-phosphate dehydrogenase. Biol. Pharm. Bull., 33, 379-383.

Takechi, S., Yamaguchi, T., Nomura, H., Minematsu, T. and Nakayama, T. (2004): Growth inhibition and mutagenesis induced in Escherichia coli by dihydropyrazines with DNA strand-cleaving activity. Mutat. Res., 560, 49-55.

Takeda, O., Takechi, S., Ito, S., Omori, H., Katoh, T. and Yamaguchi, T. (2007): Effects of phenyl derivatives of dihydropyrazines with ability to generate radical species on Escherichia coli. Biol. Pharm. Bull., 30, 1663-1667.

Takeda, O., Takechi, S., Katoh, T. and Yamaguchi, T. (2005): The role of dihydropyrazines in accelerated death of Escherichia coli 
Dihydropyrazine-induced glutathione imbalance in HepG2 cells

on addition of copper(II). Biol. Pharm. Bull., 28, 1161-1164.

Teixeira, P.C., Onuki, J., Medeiros, M.H., Dörnemann, D. and Di Mascio, P. (2001): DNA damage by 3,6-dihydropyrazine-2,5dipropanoic acid, the cyclic dimerization product of 5 -aminolevulinic acid. Biol. Chem., 382, 913-918.

Yamaguchi, T., Eto, M., Harano, K., Kashige, N., Watanabe, K. and Ito, S. (1999): New compounds derived from dihydropyrazines having DNA strand-breakage activity. Tetrahedron, 55, 675-686.

Yamaguchi, T., Ito, S., Kashige, N., Nakahara, K. and Harano, K. (2007): The relationship between the chemical structures of dihydropyrazine derivatives and DNA strand-breakage activity. Chem. Pharm. Bull., 55, 532-536.
Yamaguchi, T., Kashige, N., Mishiro, N., Miake, F. and Watanabe, K. (1996): Dihydropyrazine derivatives as a new type of DNA strand breaking agent. Biol. Pharm. Bull., 19, 1261-1265.

Yamaguchi, T., Matsumoto, S., Masumizu, T., Takechi, S., Ishida, T., Takeshita, K., Kansui, H. and Kunieda, T. (2012): Generation of radical species from dihydropyrazines having DNA strandbreakage activity and other characteristics. Chem. Pharm. Bull., 60, 639-646.

Zlatkis, A., Bertsch, W., Lichtenstein, H.A., Tishbee, A., Shunbo, F., Liebich, H.M., Coscia, A.M. and Fleischer, N. (1973): Profile of volatile metabolites in urine by gas chromatography-mass spectrometry. Anal. Chem., 45, 763-767. 